$\xi=-1$

\title{
Shared intelligent optimum route selection through traffic management system in VANET-SIRS
}

\author{
Mukund B Wagh ${ }^{1 *}$, N. Gomathi ${ }^{2}$ \\ ${ }^{1}$ Research Scholor, Departement of Computer Scince \& Engineering, VelTech Dr. RR and Dr. SR University, Chennai, India \\ ${ }^{2}$ Professor, Departement of Computer Scince \& Engineering, VelTech \\ *Corresponding author E-mail: mukund.wagh.81@gmail.com
}

\begin{abstract}
A Vehicular Ad-hoc Network (VANET) consists of the vehicle to vehicle and vehicle to roadside communication. Growing the density of the vehicles on the road causes flooding while transferring packets from a source to one or multiple destinations over a VANET. However, increasing vehicles on road may causes a problem such as redundancy, congestion, collision and increasing delay though receiving emergency warnings. As long as reliable and proficient multi-hop broadcast in vehicular ad hoc networks is challenging one. In the proposed system commence a new protocol which uses the character of elegant route establishment and selection method for transfer information to the destination (dst) with minimum redundancy, delay and highest bits per second delivery. In some environment vehicles are deployed in a dense manner, and it can move at a high speed. Adjacent Access Points (APs) on the road find out route information from their past report gatherings which use a fuzzy logic to select routing conclusion. This approach consists of isolating the whole network area into clusters. Based on which an optimized route is chosen using the input parameters as vehicle density, distance from the adjacent AP, vehicle mobility, receiving signal strength and delay should be jointly measured in the relay node selection. Though, these computation conflicts with each other depend on vehicle mobility. Hence, we employ fuzzy logic to handle this imprecise information to select forwarding vehicles by mutually taking into account of vehicle distance (d), mobility (v) and Receiving Signal Strength (RSS). The selected vehicle can provide an assure data forwarding with a high competence. The performance of the proposed scheme is evaluated on the metrics such as end-to-end delay, packet delivery ratio by varying vehicle speed, and density of vehicles.
\end{abstract}

Keywords: Fuzzy Optimized Forward Node Selection; Performance evaluation; Routing; Redundancy; Vehicular Sensor Networks (VSNs).

\section{Introduction}

Information sharing is essential everywhere including vehicles on the road. One of the problems so far addressed is how to resolve the possible fitness of vehicles most likely to convey in the shortest time. To optimize the traffic and to preserve human lives, vehicles need to exchange information on road regarding traffic conditions. The essential condition for the broadcasting of this information should deliver to the destination as soon as possible which provides massive benefits for road safety, publics comfort and traffic effectiveness. The traveler in the vehicles has information as regards the outside situation relating to the parameters such as the density of traffic, current location, and the map of the sites to be visited, the amount

of fumes outside the vehicles. Due to the high density of the road, vehicle movements and fading of wireless communication provides a reliable and capable multi-hop broadcast in VANET is still a challenging topic. Road traffic information used to select the optimized route, however because of the frequent changes in mobility, incorrect route can computed. Increase in the traffic density of road mainly in urban area, it would be a sturdy problem to route the packets because of congestion. Thus, there is an optimized way, which is adaptive with respect to the topological changes due to the rapid speed of the vehicle thus it generates alert warnings. Observation of the above-mentioned disputes in the VANET's; proposed a shared intelligent optimum routing strategy that can help the rescue actions (a). In the proposed approach, a shared intelligent knowledge observes from its environment and finds the vehicle density and distance from Road Side Units (RSUs). These observations are shared with the adjacent vehicles in a broadcast method. The selection of a route depends upon the consideration of the inter-vehicle distance(d), vehicle mobility(v) and Receiving Signal Strength (RSS) should be together measured in the next hop node selection from the destination in that area. This model considers Vehicle to Infrastructure (V2I) and Vehicle to Vehicle (V2V) communication. Though, this consideration value difference with each vehicle depends on mobility, and wireless channel fading condition. Best next hop selection is complex to obtain and choose result with variance values would be an expensive one. Therefore, proposed design uses fuzzy logic to handle these unsure values. Proposed system use a fuzzy logic to select next hop nodes by mutually considering d, v and RSS. The selected next hop vehicle can provide a reliable data forwarding with a high efficiency.

This paper is organized as segment II confer related work of VANET. Segment III explains the environment preface to know the proposed concept. Segment IV presents the network formation with a problem statement. Segment V describes the proposed approach and the algorithm. The simulation results with discussion are presented in Section VI. Finally, Section VII concludes this paper with directions for future work. 


\section{Literature review}

\subsection{Related work}

This paper introduced several new security features to reduce the risk of taxi crimes and to preserve the privacy of taxi passengers. The delay in waiting time is introduced by the security measures is only marginal and the amount of data to be transmitted by the scheme is acceptable [8]. Allow vehicles to generate provably anonymous and computationally well-organized pseudonyms to ensure conditional privacy. PACP does not rely on storing multiple pseudonym certificates issued from a centralized authority or on providing identity certificates to the RSU for generating on-the-fly pseudonym certificates. Instead, a node generates its pseudonyms with assistance from the RSU in its neighborhood in a way that the RSU gains no information about the node's real identity may increase computational cost [5]. Genetic algorithm is used to find the optimal path from source and destination node [12].

A Vehicular Ad-hoc Network (VANET) is considered as a specific kind of Mobile Ad-hoc Network (MANET) which consists of a set of mobile nodes (Vehicles) and fixed nodes known as Road-Side Units (RSUs)[9]. The objective of this design is to reduce tracking error and improve the robustness and performance of cooperative vehicle safety systems under different traffic conditions [1].

The concept of Encounter Probability (an estimation of the likelihood that a vehicle will meet an event) to determine whether an event is relevant enough to be rediffused or not [4] discussed. ACPN provides the conditional vehicle anonymity for privacy preservation with traceability for the non-repudiation, in case that malicious vehicles abuse anonymous authentication techniques to achieve malicious attacks [10]. Focus on VANETs in both highway and urban scenarios [6] tested.

The management of the VANET network must be for secure routing over networking most crucial is to be established [11]. Sources which can be as varied, generating huge flow of data [2] analyzed. Clustering methods can be suitable for creating scale acceptability in the network [7].

Two Cost-effective data replication algorithms are used to find the best vehicle to replicate each data item inside the platoon [3].

\section{Environment preface}

Using self-observation from environment based actions which use a fuzzy logic to enhance multi-hop route selection discussed in this protocol. By sharing environment knowledge, vehicles can take a best possible decision through the repeated contacts.

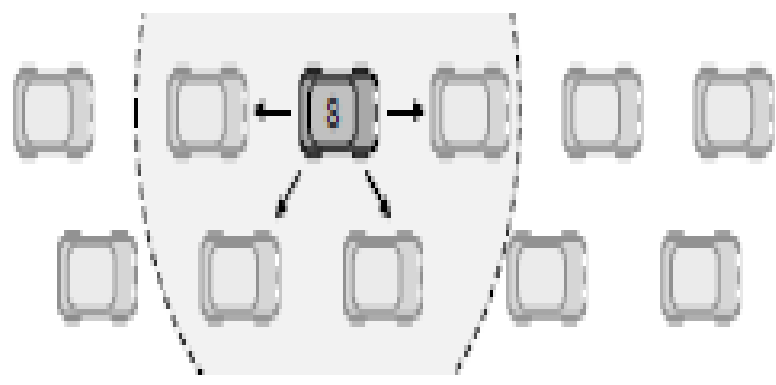

Fig. 1: Sharing Environment Knowledge.

Shared intelligent route selection and optimal time through traffic management system (SIRS) select the most stable route, by calculating the link stability and the distance of the vehicle from the sender defined as $(\mathrm{Q}, \mathrm{K}, \operatorname{Pr}, \delta, \omega)$ where

1) $Q=\left\{q_{1}, q_{2} \ldots q_{n}\right\}$ are the fixed set of event;
2) $K=\left\{k_{1}, k_{2} \ldots k_{n}\right\}$ are the fixed set of process performed by the routing vehicle;

3) $\operatorname{Pr}=\left\{\operatorname{pr}_{1}, \operatorname{pr}^{2} \ldots, \mathrm{pr}_{\mathrm{n}}\right\}$ are the fixed set of response received from the network; and

4) $\delta=\mathrm{Q}: \mathrm{X} \times \operatorname{Pr} \rightarrow \mathrm{Q}$ Maps the input of current road situation wto the next dicision through response.

Selected next hop vehicle suddenly accelerates and vary its speed, the route that follows the next vehicle will fail. Solving this issue prediction of the network constancy requires, estimation of the vehicle behavior to some extent Based on the current road situation in which the next decision can be defined as where

1) $X=\left(X_{1}, X_{2}, \ldots \ldots X_{n}\right)$ are fixed number of inputs;

2) $Y=\left(Y_{1}, Y_{2} \ldots, Y_{n}\right)$ are reinforcement signal values; and

3) $\rho=\left(\rho_{1}, \rho_{2} \ldots \rho_{n}\right)$ are punishment possibilities with each $X_{i},(1$ $\leq \mathrm{i} \leq \mathrm{n})$.

The sharing knowledge carries out a limited number of decisions based upon their response; it can be either incentive or a punishment. To predict the movements of the vehicle, observe its changes, maintain a history of them with time. Take decision using this information to avoid selecting unstable vehicles. Take each vehicle speed number of sample $\left(\mathrm{V}_{0}, \mathrm{~V}_{1}, \mathrm{~V}_{2} \ldots ., \mathrm{V}_{\mathrm{n}-1}\right)$ at time $\mathrm{t}_{0}$, $\mathrm{t}_{1} \ldots \ldots \mathrm{t}_{\mathrm{n}-1}$. As time passes, collect latest observations about the movement. A stable vehicle does not have many variations in the speed and an unstable vehicle shows lots of variations in speed and update decision probability Dp by sharing knowledge Figure 1 shows. If vehicle receives incentive from the network then update Dp. Computer $D_{p}$ update is as follows: where a is a parameter

$\operatorname{Pj}(n+1)=(1-a) P j(n), j \neq i, Y=0$

$\operatorname{Pj}(n+1)=\operatorname{Dpj}(n), j=i, Y=0$

$P j(n+1)=P j(n), Y=1$

\section{Network formation}

The network consists of $n$ number of vehicles with a unique ID build with a Global Positioning System (GPS), broadcasting antenna, camera, and storage space. Road side network equipments are connected via gateway to the Internet. Road Side Units (RSU) or Access Points (AP) is deployed in various places of the road shows in Figure 2. The RSU send or receive the information between gateway and vehicles. In Vehicle to Vehicle (V2V) communication, each vehicle is in touch with the other vehicle directly in the communication range. Divide the road into clusters in a single row. Figure 3 shows the road infrastructure. While network density enhanced vehicle speed reduced due to congestions and collision.

\subsection{Problem definition}

Let $\mathrm{P}=\{\mathrm{P} 1, \mathrm{P} 2 \ldots \mathrm{Pn}\}$ be the current position of the vehicles; $\mathrm{S}=$ $\left\{S_{1}, S_{2} \ldots . . S n\right\}$, the space from the neighboring $R S U ; Q=\left\{Q_{1}\right.$, $\left.\mathrm{Q}_{2} \ldots \mathrm{Qn}\right\}$ the vehicle quantity set; and $\mathrm{R}=\left\{\mathrm{R}_{1}, \mathrm{R}_{2} \ldots \mathrm{Rn}\right\}$ the current routes set. Using GPS current position of the vehicle can be obtained. To make a change from one state to another, switching function as defined as:

$\delta_{\mathrm{ij}}: \mathrm{X} \rightarrow \mathrm{R}$

Where $\mathrm{X}=(\mathrm{P}, \mathrm{S}, \mathrm{Q})$ are input parameters, $1 \leq \mathrm{i} \leq \mathrm{n}, 1 \leq \mathrm{j} \leq \mathrm{n}$. $\mathrm{R}$ has the potential routes, and $\delta_{\mathrm{ij}}$ is a stochastic function, which acts based on input parameters. It maps the present position of the vehicle, distance from the RSU and the appropriate route vehicle density. The vehicle density is measured by the number of vehicles in a cluster to the total number of vehicles computed as

$D=\frac{\sum_{i=1}^{n} N V i}{\sum_{i=1}^{n} n i}$ 


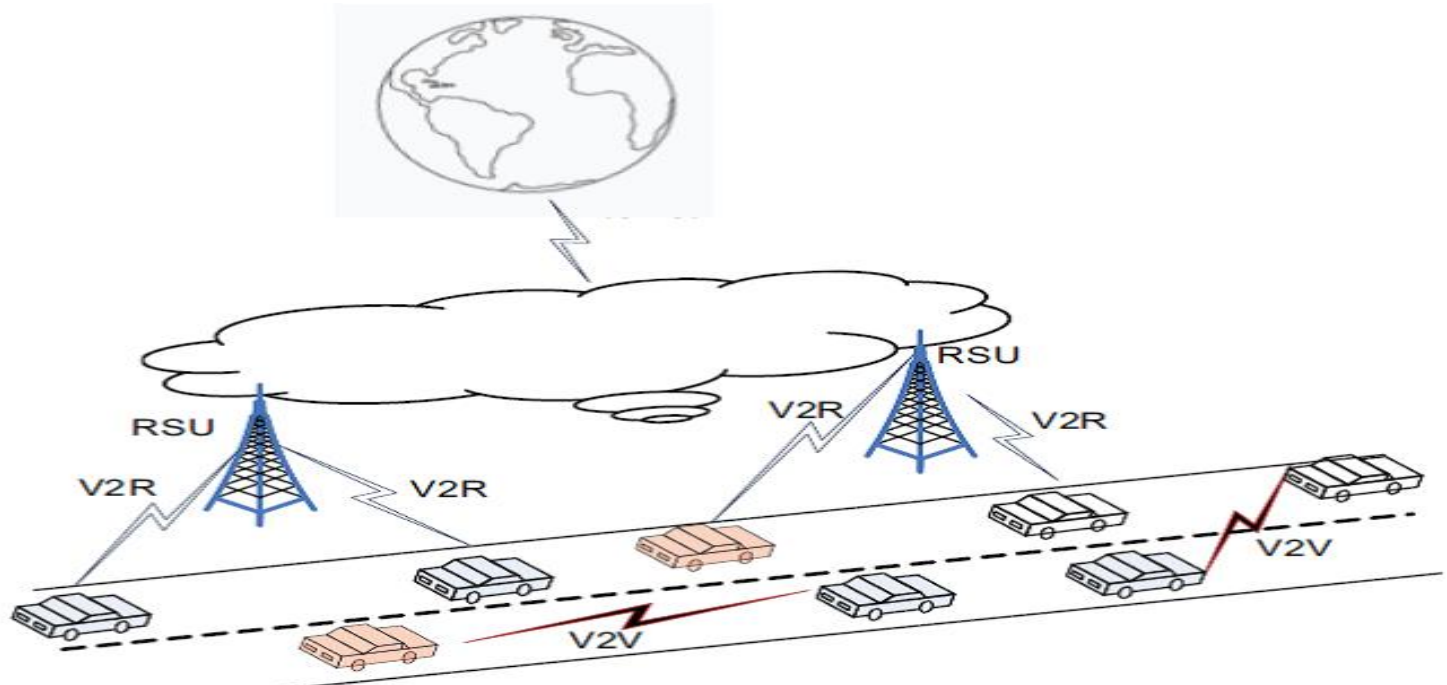

Fig. 2: Proposed System Model.

Number of vehicles in the cluster (NVi) and a total number of vehicles on the road ni. Set a predefined range of 250 meters as a cluster region to monitor the vehicles. Each cluster has different density of vehicles; routing mapped with input parameters and the present route using the values in Switching Function Matrix (SFM) describe as in the following. Computed values furnish the possibility of for route selection SFM computed as follows:

$$
\left[\begin{array}{l}
\partial_{11} \partial_{12}----\partial_{1 n} \\
\partial_{2} \partial_{n 2}----\partial_{2 n} \\
-------- \\
\partial_{n} \partial_{n 2}----\partial_{m}
\end{array}\right]
$$

Where $\delta_{\mathrm{ij}}$ calculated by equation (1). Hence, protocol finds the optimized route with minimum weight with different parameters.

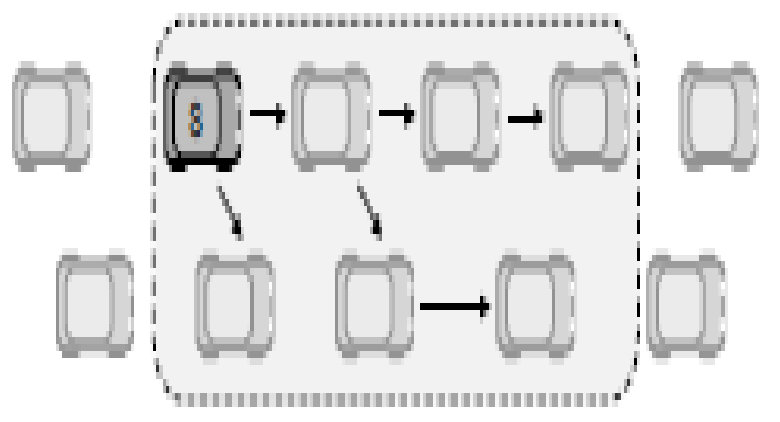

Fig. 3: Density of the Network.

\section{Proposed methodology}

Shared Intelligent Optimum Route Selection through Traffic Management System in VANET- SIRS. SIRS executes the route formation and route selection segments. The route formation phase consists of the construction of routes using sharing knowledge, although the route selection consists of smart route selection.

\subsection{Route establishment (RE)}

Source (s) starts routing packet has vehicle current position, distance from RSUs vehicle quantity, total delay $\delta$ t, transmit to the intermediate vehicle. Upon vehicle decides its next movement. Route consistency is resulted from vehicles Link stability. The excellence of fuzzy estimation depends on the quality of the fuzzy rules. The fuzzy logic projected to check the Link constancy (LC) of each link between sender and receiver. The three input variables to be fuzzified are d, v, RSS of the neighbor vehicles. The inputs are fuzzified, and defuzzified to get the sharp value of LC as the output. The linguistic variables connected with the input variables are small (s), medium (m) and (l) large. For the output variable, Speed considered as SLOW, MEDIUM, FAST, distance taken as LESS, MEDIUM, HIGH and RSS verified as BAD, MODERATE, And GOOD. Here, we use LCi, $\mathrm{j}$ denote the LC between vehicle $\mathrm{i}$ and $\mathrm{j}$. Assume one communication route between source and destination is made up of $\mathrm{n}$ intermitted nodes.

RCPs, d = LCs, $1 *$ LC1 1, $2 *$ LC2, $3 * \ldots *$ LCn, d RCPs, d denotes the Route constancy pointer of the whole route. After selecting next vehicle based on defuzzification the route from $\mathrm{s}$ to dst launched based on the switching function $\delta_{\mathrm{ij}}$. The $\delta_{\mathrm{i}}$ values adjust as per the incentive, punishment also $D_{p}$ decides based on the threshold (THR) for each path which can be able to finalizes the packet transmission capacity of that route.

$$
T H R_{i}=\left(1 / n_{-} p\right) \sum_{i=1}^{n} \sum_{W_{i}(t)}
$$

$n \_p$ is the number of route from $s$ to dst and $W_{i}(t)$ is the growing weight of these routes computed as:

$$
\overline{w_{i}(t)}=\left(1 / N\left(n_{-} p\right)\right) \sum_{i=1}^{N\left(n_{n} p\right)} X_{i}^{2}
$$

Here $N$ (n_p) is the number of times weight along that route has been computed; also $\mathrm{W}$ (j) $t$ is the weight of each route decides the incentive and punishment. If the vehicle is dst with reward, then update growing weight with $\psi \in[0,1]$ as a reward values, the high value specifies the best route selection. Switching function also used for update the growing weight. Punishment is connected, as $\varphi \in[0,1]$ is the punishment limitation, it reduces the growing weight for each failed transmission. Where $\varphi$ is used to reduce the result of punishment. Vehicle chooses or discards a packet as per THRw. If current road report has higher distance and density than the defined THRi than the previous report, reject new packet otherwise, update the route for the final dst for future communication.

\subsection{Route Selection (RS)}

After route establishment selects next hop from the routing table maintained by each vehicle. Each vehicle selects its best neighbor, which has the best fuzzy value, so that packets can be transferred to the neighbor vehicle.

Gather road state at different time intervals about vehicle $\mathrm{v}, \mathrm{d}$ and density from the final destination. At next interval, vehicle speed 
may vary as per road density. Sharing knowledge at each time interval gives best path selection. Let $\mathrm{v}$ be the speed of the vehicle at time $t$ and $v \pm d v$, its speed in the next interval is $t+d t$ Let $\operatorname{Ak}(n+1), A k(n), A_{l}(n+1), A_{1}(n)$ be the various report states at different time periods. If newly arrived report has higher density than the current report, select the next destination based on the low density and high value of entries from SFM. All roads divided into number of clusters, so compute vehicle probability in a particular cluster at time t.

This information is shared by each vehicle; it will then have less chance of collision and, at the same time, higher chances of packet transmission to the desired destination. Probability may vary as per road environment and cluster changes. Thus, the change in the density of the vehicle as $\operatorname{Ak}(n+1), \operatorname{Ak}(n), A_{l}(n+1), A_{l}(n)$. The value of $\Delta \mathrm{D}$ is dependent on the vehicle arrival rate and their mean time to stay in a particular cluster.
If the newly calculated density and distance from the RSU are more than the old, then arrived packet at the intermediate node is discarded; otherwise, the packet is kept as a repository for future. Moreover, if this value is less than the old value, then the routing table is updated by adding this new value and all the previous paths are discarded.

\section{Experimental results}

Figure 4 describes the output of increasing vehicle density based on the probability of safety alert message transfer to the destination. When node density increases, the probability of alerts transfer decreases to its final destination. The vehicle takes the decisions using fuzzy with nodes past behavior and safety alert message transfer to destination increased.

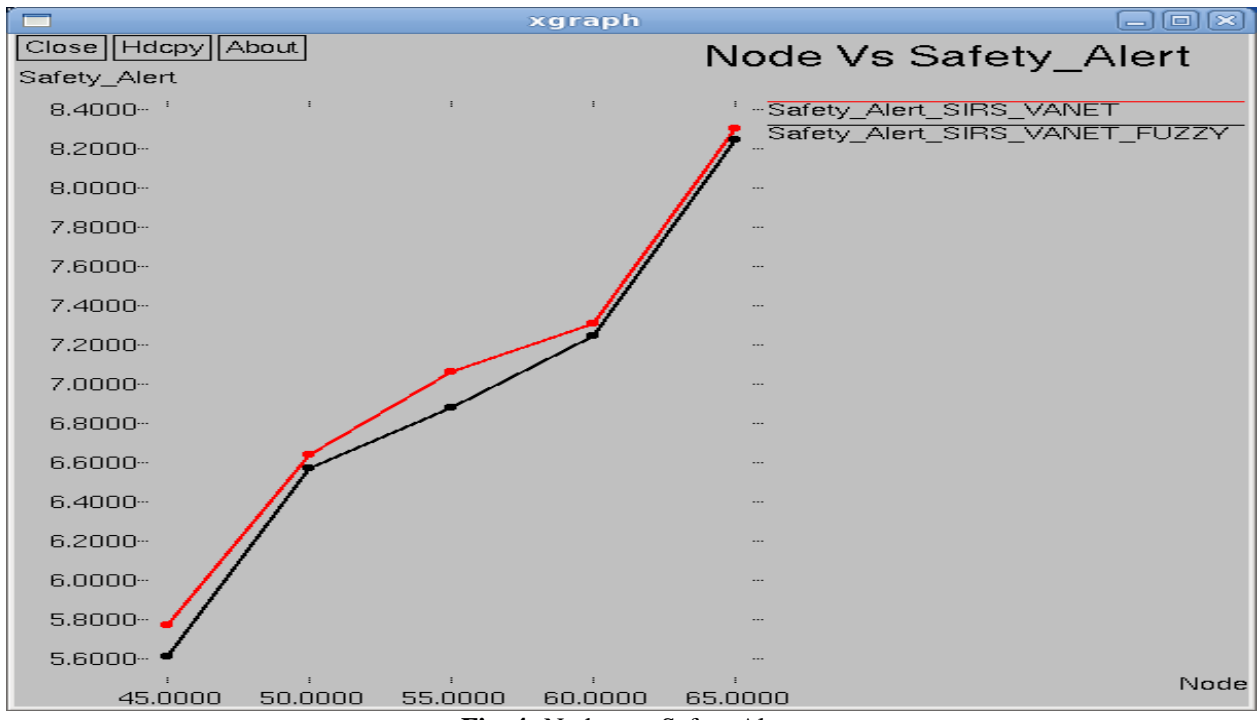

Fig. 4: Nodes vs. Safety Alert.

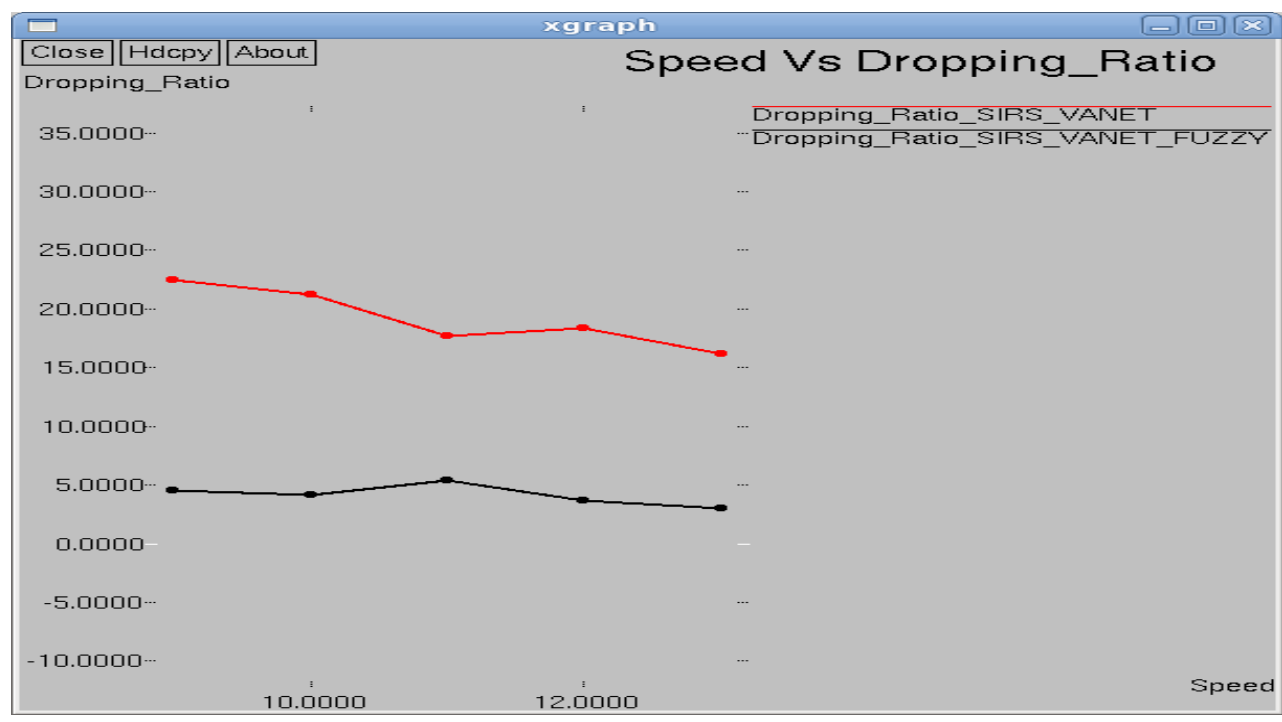

Fig. 5: Speed vs. Dropping Ratio. 


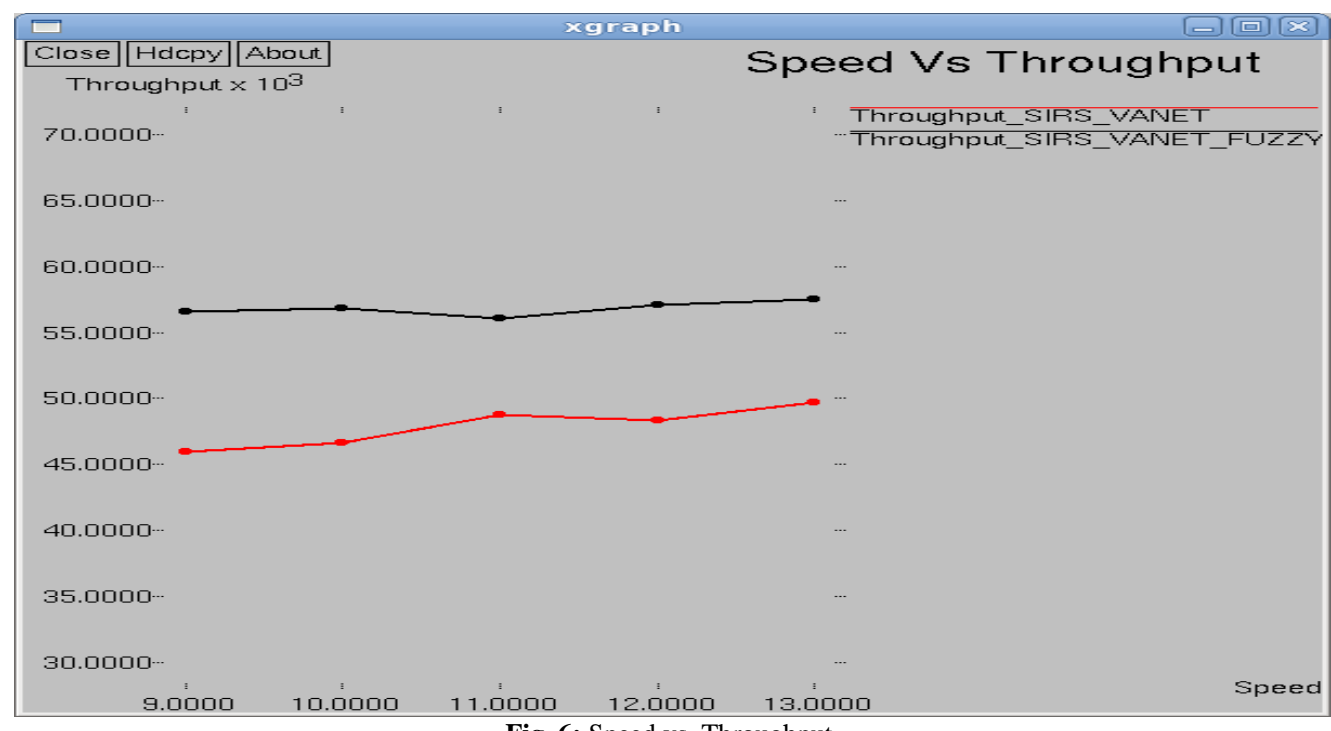

When frequent network disconnection occurs, the result of packet drop ratio increased. This drop ratio helps to find routing selection changes. Density increasing, congestion, communication failure leads increasing packet drop ratio.

Figure 5 shows reducing packet loss with fuzzy computation by Number of lost packets / Number of received packets.

Throughput is the number of transmissions that a vehicle completed in the given time period. Basically, throughput has been calculated in the dense network that may run huge volume of transmissions continuously. Fig.6 illustrates that gradually increasing throughput by fuzzy logic.

Throughput $=$ pkts $* 8 /$ data duration.

\section{Conclusion}

VANET networks are designed with dynamic network changes, unassuming traffic, and different Contexts need a novel method of routing described in SIR's with fuzzy that can help in the urban dense areas at critic situations. This approach provides stable routing using fuzzy logic with computation of delay, distance of vehicle and RSU, mobility, traffic, and RSS. So that the protocol gives best result in VANET communication also supports to select effective stability when selecting routes using decision-making technique of fuzzy logic.

\section{References}

[1] Ching-Ling Huang, Yaser P. Fallah, and Raja Sengupta," Adaptive Intervehicle Communication Control for Cooperative Safety Systems"IEEE Network January/February 2010. https://doi.org/10.1109/MNET.2010.5395777.

[2] S. Raghay, S. El Hadaj \& T. Noe, "A Genetic Algorithm for Management Data Stream in Vanet'International Journal of Information Technology and Knowledge Management January-June 2011, Volume 4, No. 1, pp. 315-318.

[3] Yang Zhang, Student Member, IEEE, and Guohong Cao,"V-PADA: Vehicle-Platoon-Aware Data Access in VANETs"IEEE TRANSACTIONS ON VEHICULAR TECHNOLOGY, VOL. 60, NO. 5, JUNE 2011.

[4] Nicolas Cenerario, Thierry Delot, and Sergio Ilarri, "A ContentBased Dissemination Protocol for VANETs: Exploiting the Encounter Probability"'IEEE TRANSACTIONS ON INTELLIGENT TRANSPORTATION SYSTEMS, VOL. 12, NO. 3, SEPTEMBER 2011.

[5] Dijiang Huang, Senior Member, IEEE, Satyajayant Misra, Member, IEEE, Mayank Verma, Member, IEEE, and Guoliang Xue, Fellow, IEEE,"PACP: An Efficient Pseudonymous Authentication-Based Conditional Privacy Protocol for VANETs" IEEE TRANSAC-
TIONS ON INTELLIGENT TRANSPORTATION SYSTEMS, VOL. 12, NO. 3, SEPTEMBER 2011.

[6] Francisco Javier Ros, Pedro Miguel Ruiz, Member, IEEE, and Ivan Stojmenovic,"Acknowledgment-Based Broadcast Protocol for Reliable and Efficient Data Dissemination in Vehicular Ad Hoc Networks"IEEE TRANSACTIONS ON MOBILE COMPUTING, VOL. 11, NO. 1, JANUARY 2012.

[7] Mehrnaz Mottahedi, Sam Jabbehdari and Sepideh Adabi, "IBCAV: Intelligent Based Clustering Algorithm in VANET'IJCSI International Journal of Computer Science Issues, Vol. 10, Issue 1, No 2, January 2013.

[8] T.W. Chim, S.M. Yiu , Lucas C.K. Hui , Victor O.K. Li, “VANETbased secure taxi service"VANET-based secure taxi service, Ad Hoc Netw. (2013). https://doi.org/10.1016/j.adhoc.2013.06.003.

[9] Salim Bitam, Abdelhamid Mellouk, Senior Member, IEEE, and Sherali Zeadally, Senior Member,"Bio-Inspired Routing Algorithms Survey for Vehicular Ad Hoc Networks"IEEE COMMUNICATION SURVEYS \& TUTORIALS, VOL. 17, NO. 2, SECOND QUARTER 2015.

[10] Jie Li, Senior Member, IEEE, Huang Lu, Member, IEEE, and Mohsen Guizani, "ACPN: A Novel Authentication Framework with Conditional Privacy-Preservation and Non-Repudiation for VANETs"IEEE TRANSACTIONS ON PARALLEL AND DISTRIBUTED SYSTEMS, VOL. 26, NO. 4, APRIL 2015.

[11] Rajinder Kaur, Dr. Shashi B. Rana, "Overview on Routing Protocols in VANET'International Research Journal of Engineering and Technology (IRJET), Volume: 02 Issue: 03 | June-2015.

[12] Shabad Kaur, Amandeep Kaur Virk, "AODV Extension Using Genetic Algorithm in VANET"International Journal of Science and Research (IJSR), Volume 4 Issue 7, July 2015.

[13] Marcin Seredynski, Gregoire Danoy, Masoud Tabatabaei, Pascal Bouvry and Yoann Pignne, "Generation of Realistic Mobility for VANETs Using Genetic Algorithms", IEEE Congress on Evolutionary Computation| June-2012. https://doi.org/10.1109/CEC.2012.6252987. 